\title{
Factors Affecting Successful Vaginal Birth Following Dinoprostone Administration in Post-term Pregnancies
}

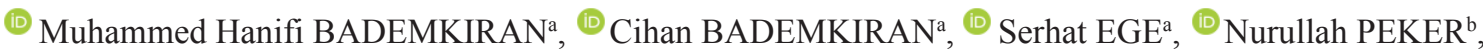 \\ (D) Süleyman Cemil OĞLAK ${ }^{\mathrm{a}}$
}

aDepartment of Obstetrics and Gynaecology, University of Health Sciences Gazi Yaşargil Training and Research Hospital, Diyarbakır, TURKEY ${ }^{b}$ Department of Obstetrics and Gynaecology, Dicle University Faculty of Medicine, Diyarbakır, TURKEY

\begin{abstract}
Objective: This study will determine the success rates of the predictive factors of vaginal birth in the post-term labor induction of the cervical ripening slow-release insert dinoprostone. Material and Methods: All patients who underwent labor induction were post-term pregnant patients. Post-term pregnancy was defined as lasting $\geq 42+0$ weeks of gestation. Dinoprostone insertion into the posterior fornix was performed according to the Bishop score $(\leq 6)$ and maintained for a maximum of 24 hours. Parity, Bishop score, maternal age, fetal gender, and induction time were identified as candidate predictors. The traditional logistic regression method was used to examine the relationship between the outcome and candidate predictors. Discrimination in the model was evaluated by calculating the c-index. Results: Of the 25,678 deliveries that occurred during the study period, 361 (1.4\%) women underwent post-term delivery; of these, 293 (81\%) succeeded, and 68 (19\%) failed to achieve cervical ripening using the dinoprostone slow-release vaginal insert. Three predictors were strongly associated with dinoprostone vaginal delivery success: multiparity (2.88[1.38-6.01]), fetal gender (1.69[0.9-3.0]), and Bishop score (OR: 1.59 [1.45-1.70]). Conclusion: The success of vaginal delivery can be predicted by evaluating factors, including fetal gender, parity, and the Bishop score in post-term pregnancies. Including these factors in the management protocol for labor induction with cervical ripening could improve care quality.
\end{abstract}

Keywords: Dinoprostone slow-release vaginal insert; post-term delivery induction; success rates; prostaglandin E2; cervical ripening; bishop score

Adverse perinatal outcomes increase gradually from the $40^{\text {th }}$ week of pregnancy, and this increase is evident significantly from the $42^{\text {nd }}$ week of pregnancy. ${ }^{1}$ With the prolongation of pregnancy, the risk of stillbirth increases, and $14 \%$ of stillbirth occur in prolonged pregnancies worldwide. ${ }^{2}$ The World Health Organization recommends inducing labor at week 41, and many countries induce labor from 41 to 42 weeks to prevent prolonged pregnancies. ${ }^{3,4}$ There are randomized controlled studies in the literature comparing the induction of labor with the expectant control group in the post-term pregnancies. In most of them, conflicting results were found in terms of perinatal morbidity and mortality. The Cochrane review in 2018 showed that the rate of cesarean delivery and perina- tal mortality was lower, and the ratio of assisted vaginal delivery was higher in the induction group compared to the control group. ${ }^{5}$

Labor induction is one of the most common interventions in the labor process. This procedure, which has fetal and obstetric indications, aims to achieve cervical ripening and stimulate the contraction of the uterus before labor. ${ }^{6}$ There are pharmacological (prostaglandins) and non-pharmacological methods (i.e., amniotomy, mechanical methods, including extra-amniotic foley catheter or the Cook cervical ripening balloon) of labor induction. Prostaglandins are the most commonly used induction agent and can be used for this purpose via different administration routes, such as orally,

Correspondence: M. Hanifi BADEMKIRAN

Department of Obstetrics and Gynaecology, University of Health Sciences Gazi Yaşargil Training and Research Hospital, Diyarbakır, TURKEY

E-mail: hanifibademkiran@hotmail.com

Peer review under responsibility of Journal of Clinical Obstetrics \& Gynecology. 
intravenously, extra-amniotic, vaginal, or intracervical. ${ }^{7}$ Since many factors predict the success or failure of the labor after applying prostaglandins, it is not easy to find the main factor. ${ }^{8}$ There are few studies on the factors affecting successful labor induction with dinoprostone in post-term pregnancies. ${ }^{9,10}$

This retrospective study was aimed to determine the success rates of the predictive factors of vaginal birth in the post-term labor induction of the cervical ripening slow-release insert dinoprostone.

\section{MATERIALS AND METHODS}

\section{PATIENT CHARACTERISTICS}

In this retrospective study, a total of 361 single postpregnant women (usually $>=42$ weeks of gestation) who applied to Obstetrics and Gynecology Clinic, University of Health Sciences Diyarbakır Gazi Yaşargil Training and Research Hospital between September 2017 and August 2018 were included. All procedures used in studies involving human participants were approved by Diyarbakır Gazi Yaşargil Training and Research Hospital's institutional ethics review board (standard no: 02/2018-21) and conducted in accordance with the Helsinki Declaration of 1964 and its subsequent amendments or comparable ethical standards.

Patient data were collected from the comprehensive perinatal database in maternal-demographic and obstetric characteristics data, including parity, maternal age, hypertension, and diabetes mellitus (DM). Clinical features included Bishop score, gestational age, delivery mode, cause of cesarean section, postpartum hemorrhage, intrapartum complications, fetal gender, birth weight, and neonatal outcomes (1-min Apgar score and 5-min Apgar score). All patients who underwent labor induction were post-term pregnant patients. Post-term pregnancy was defined as lasting $\geq 42+0$ weeks of gestation. Gestational age was measured by the first day of the patient's last menstrual period and/or firsttrimester ultrasound measurements.

\section{PROCEDURES}

According to the department protocol, women undergoing induction were evaluated by vaginal exam- ination (cervical position, cervical dilation, cervical effacement, cervical consistency, and fetal presentation part station) to achieve Bishop scores. Intrapartum fetal heart rate monitoring was performed before the dinoprostone (PROPESS ${ }^{\circledR}$, Ferring, Turkey) was placed for one hour. Placement of the dinoprostone was done according to Bishop score $(\leq 6)$. It was continued for a maximum duration of 24 hours. Dinoprostone was removed in the case of painful uterine contraction, unreliable fetal heart rate, or uterine tachysystole ( $>5$ uterine contractions within 10 minutes), and the Bishop score did not change (24-hour intervals). The time from induction to delivery was defined as when PROPESS was added to the delivery time. Induction failure was defined as the inability to reach the active phase of labor (cervical dilatation of $4 \mathrm{~cm}$ or more with regular uterine contractions). We described the failure to progress as the failure of the cervix to dilate $0.5 \mathrm{~cm} / \mathrm{h}$ in the active phase or stalled the fetal head descending in the second stage of labor.

\section{STATISTICAL ANALYSIS}

In the presentation of numerical variables, the median and interquartile range and the percentage and $\mathrm{n}$ are used to present categorical variables. Student's t-test and Mann-Whitney U test were used to compare continuous variables with and without normal distribution, and the $\chi^{2}$ test was used for categorical variables. In the study, consecutive patients were evaluated according to clinical outcomes. The success of dinoprostone vaginal delivery was determined as the outcome of the clinical prediction model of this study. Parity, Bishop score, maternal age, fetal gender, and induction time were defined as candidate predictors. As a result, four candidate variables were included in the final model. This study used a multivariable logistic regression analysis to examine the relationship between the outcome and candidate predictors while controlling potential confounders. Discrimination in the model was evaluated by calculating the c-index. Statistical significance was defined as $p<0.05$. All statistical analyses were described via $\mathrm{R}$ version 3.5.1 (R Statistical Software, Institute for Statistics and Mathematics, Vienna, Austria). 


\section{RESULTS}

During the study period, a total of 25,678 pregnancies were born at our centre, of whom 361 (1.4\%) underwent preterm delivery were eligible for the study. These included 293 (81\%) succeeded, and 68 (19\%) failed to achieve cervical ripening using the dinoprostone. Maternal-demographic and obstetric characteristics of the study groups are summarized in Table 1 and Table 2.

The researchers created a model using multivariable logistic regression analysis with candidate predictors. The odds ratios (OR) and 95\% confidence intervals (CI) obtained from the model are summarized in Table 3. Three predictors were strongly connected with dinoprostone vaginal delivery success: multiparity $(2.88[1.38-6.01])$, fetal gender (1.69[0.9-3.0]), and Bishop score (OR: 1.59 [1.45-1.70]). The multivariable logistic regression model correctly classified $76 \%$ of the contributors (c-index: 0.76).

\section{DISCUSSION}

In this retrospective study, the factors determining the success of labor induction with dinoprostone vaginal insert system in post-term pregnancies were associated with fetal gender, Parity, and the Bishop score. The success of vaginal delivery can be predicted by evaluating factors, including fetal gender, parity, and the Bishop score in post-term pregnancies.

The Bishop score still exists in the vast majority of protocols to assess cervical conditions before IOL, despite many studies showing its limitations as a predictive tool ${ }^{11}$. We evaluated its performance in our population and included it in the modeling process. Not only did we find that it was related to the outcome, but it also had a predictive capacity. The

\begin{tabular}{|c|c|c|c|}
\hline Demographic characteristics & $\begin{array}{l}\text { Vaginal delivery } \\
(n=293) 81 \%\end{array}$ & $\begin{array}{l}\text { Cesarean section } \\
\quad(n=68) 19 \%\end{array}$ & p-value \\
\hline Maternal age (year), (median [IQR]) & $25(21-28)$ & $24(21-31)$ & 0.9 \\
\hline Gravida, (median [IQR]) & $2(1-2)$ & $1(1-2)$ & 0.03 \\
\hline Bishop score at induction (median [IQR]) & $3(2-4)$ & $3(2-3)$ & 0.01 \\
\hline Hypertension disorders (hypertension and preeclampsia), \% (n) & $0(0)$ & $12(8)$ & \\
\hline Gestational diabetes, $\%(n)$ & $0(0)$ & $0(0)$ & \\
\hline
\end{tabular}

Note. Values are presented as $\%(n)$; median (interquartile) bold values represent $p<0.05$.

\begin{tabular}{|c|c|c|c|}
\hline Obstetrical Characteristics & Vaginal delivery $(n=293) 81 \%$ & Cesarean section $(n=68) 19 \%$ & p-value \\
\hline 1-min Apgar score & $7.8(6-9)$ & $7.5(6-9)$ & 0.0002 \\
\hline 5-min Apgar score & $8.9(8-10)$ & $8.7(8-10)$ & 0.001 \\
\hline No. of prostaglandin E2, \% (n) & & & 0.001 \\
\hline 1 & $99(292)$ & $79(54)$ & \\
\hline 2 & $1(1)$ & $21(14)$ & \\
\hline Fetal weight (gram), (median [IQR]) & $3400(3150-3650)$ & $3400(3200-3700)$ & 0.4 \\
\hline \multicolumn{4}{|l|}{ Reason for cesarean section } \\
\hline Induction failure, \% (n) & $6(4)$ & & \\
\hline Failure to progress, $\%$ (n) & $59(40)$ & & \\
\hline Non-reassuring fetal heart rate, $\%$ (n) & $29(20)$ & & \\
\hline Aggravation of maternal state, $\%$ (n) & $6(4)$ & & \\
\hline
\end{tabular}

Note. Values are presented as $\%(n)$; median (interquartile) bold values represent $p<0.05$. 


\begin{tabular}{|lcc|}
\hline \multicolumn{2}{|c|}{ TABLE 3: Penalized adjusted odds ratios to track the association of candidate factors with the success of post-term } \\
& \multicolumn{1}{|c|}{ dinoprostone induction. } \\
\hline Variables & OR, 95\% Cl & p-value \\
Maternal age & $0.95(0.90-1.0)$ & 0.08 \\
Bishop score at induction & 1.00 (referent) & 0.08 \\
Bishop score $=1$ & $1.83(0.9-3.62)$ & 0.01 \\
Bishop score $=2$ & $2.75(1.23-3.62)$ & 0.08 \\
Bishop score $>=3$ & $1.69(0.9-3.0)$ & \\
Fetal gender & & 0.004 \\
Parity & $1.00($ referent) & 0.0001 \\
Nulliparity & $2.88(1.38-6.01)$ & \\
Multiparity & $0.89(0.86-0.93)$ & \\
Induction time & & \\
\hline
\end{tabular}

Bishop score is an important variable, and it has resulted in being predictive as an independent variable in most of the previous studies. ${ }^{12,13}$ Kolkman et al. believed that Bishop score is not strong enough as a predictive factor in deciding whether to induce a patient or not and therefore, they recommend not using it for this purpose. ${ }^{14}$ In our study, we determined that the Bishop score was a useful variable in predicting the induction success of dinoprostone. Therefore, the Bishop score can be used as a predictive factor in the success of dinoprostone before labor induction.

Parity was significantly correlated with successful induction. ${ }^{15}$ Parity is an essential predictor in many studies. ${ }^{16-18}$ Nulliparity, as expected, was a significant predictor for cesarean delivery following induction of labor even after adjusted analysis. ${ }^{16}$ We found it to be the most important predictor of successful induction. In our predictive modeling, when nulliparity was determined as the reference point, we discovered that multiparity was 2.8 times more predictive in induction success with dinoprostone.

There are a few studies on the effect of fetal gender on predicting the success of labor induction. ${ }^{19,20}$ It was stated that the male gender is a predictive factor in patients delivered by cesarean section. ${ }^{19}$ In our predictive modeling, we demonstrated that the female gender is 1.6 times more predictive in the success of labor induction when the male gender is determined as the reference point.
As in our study, many studies create clinical predictive modeling by adding maternal and fetal characteristics. ${ }^{21,22}$ In our research, the c-index showing the performance of our modeling was found to be 0.76 . The c-index is higher than 0.75 is considered a parameter indicating that modeling's performance is effective. ${ }^{23}$

The strengths of this study include the use of a single protocol in a tertiary center with a large number of cases. The researchers acknowledge the data limitations. First of all, this is a retrospective study that may be open to selection bias. Likewise, the study lacks data on body mass index may be hiding other undetected weaknesses.

\section{CONCLUSION}

Basic parameters such as Bishop score, maternal gender, and parity can predict successful vaginal birth following the dinoprostone administration. Including these factors in the management protocol for postterm labor induction may improve patient care quality. It will assist inform the patient about induction success before post-term labor induction.

\section{Source of Finance}

During this study, no financial or spiritual support was received neither from any pharmaceutical company that has a direct connection with the research subject, nor from a company that provides or produces medical instruments and materials which may negatively affect the evaluation process of this study. 


\section{Conflict of Interest}

No conflicts of interest between the authors and / or family members of the scientific and medical committee members or members of the potential conflicts of interest, counseling, expertise, working conditions, share holding and similar situations in any firm.

\section{Authorship Contributions}

Idea/Concept: Muhammed Hanifi Bademktran, Cihan Bademkıran Design: Süleyman Cemil Oğlak; Control/Supervi- sion: Muhammed Hanifi Bademkıran; Data Collection and/or Processing: Serhat Ege, Nurullah Peker; Analysis and/or Interpretation: Muhammed Hanifi Bademkıran, Nurullah Peker; Literature Review: Süleyman Cemil Oğlak; Writing the Article: Muhammed Hanifi Bademkıran, Cihan Bademkıran; Critical Review: Serhat Ege, Süleyman Cemil Oğlak; References and Fundings: Nurullah Peker, Cihan Bademkiran; Materials: Serhat Ege, Nurullah Peker.

\section{REFERENCES}

1. Olesen AW, Westergaard JG, Olsen J. Perinatal and maternal complications related to postterm delivery: a national register-based study, 1978-1993. Am J Obstet Gynecol. 2003;189(1):222-7.[Crossref] [PubMed]

2. Lawn JE, Blencowe H, Waiswa $\mathrm{P}$, et al. Stillbirths: rates, risk factors, and acceleration towards 2030. Lancet. 2016;387(10018): 587-603.[Crossref] [PubMed]

3. WHO recommendations: Induction of labour at or beyond term. Geneva: World Health Organization; 2018.[Link]

4. American College of Obstetricians and Gynecologists. Practice bulletin no. 146: Management of late-term and post-term pregnancies. Obstet Gynecol. 2014;124(2 Pt 1):390-6. [Crossref] [PubMed]

5. Middleton $\mathrm{P}$, Shepherd E, Crowther CA. Induction of labour for improving birth outcomes for women at or beyond term. Cochrane Database Syst Rev. 2018;5(5):CD004945.[Crossref] [PubMed]

6. WHO Recommendations for Induction of Labour. Geneva: World Health Organization; 2011.[Link]

7. Leduc D, Biringer A, Lee L, Dy J; clinical practice obstetrics committee, special contributors. Induction of labour. J Obstet Gynaecol Can. 2013;35(9):840-57. [Crossref] [PubMed]

8. Batinelli L, Serafini A, Nante N, Petraglia F, Severi FM, Messina G. Induction of labour: clinical predictive factors for success and failure. J Obstet Gynaecol. 2018;38(3):352-8. [Crossref] [PubMed]

9. Modrzyńska A, Radoń-Pokracka M, Płonka M, et al. Labor induction at full-term and post-term pregnancies. Folia Med Cracov. 2019;59(4): 79-94.[Crossref] [PubMed]

10. Farina A, Bernabini D, Rapacchia G, et al. Vaginal delivery rate in post-term pregnancies with one versus more than one dinoprostone gel administrations: an observational study. Minerva Ginecol. 2013;65(5):567-75. [PubMed]

11. Migliorelli $\mathrm{F}, \mathrm{Ba}-\mathrm{os} \mathrm{N}$, Angeles MA, et al. Clinical and Sonographic Model to Predict Cesarean Delivery after Induction of Labor at Term. Fetal Diagn Ther. 2019;46(2):88-96. [Crossref] [PubMed]

12. Riboni F, Garofalo G, Pascoli I, et al. Labour induction at term: clinical, biophysical and molecular predictive factors. Arch Gynecol Obstet. 2012;286(5):1123-9. [Crossref] [PubMed]

13. Uyar Y, Erbay G, Demir BC, Baytur Y. Comparison of the Bishop score, body mass index and transvaginal cervical length in predicting the success of labor induction. Arch Gynecol Obstet. 2009;280(3):357-62.[Crossref] [PubMed]

14. Kolkman DG, Verhoeven CJ, Brinkhorst SJ, et al. The Bishop score as a predictor of labor induction success: a systematic review. Am J Perinatol. 2013;30(8):625-30.[Crossref] [PubMed]

15. Gonen R, Degani S, Ron A. Prediction of successful induction of labor: comparison of transvaginal ultrasonography and the Bishop score. Eur J Ultrasound. 1998;7(3):183-7.[Crossref] [PubMed]

16. Rane SM, Guirgis RR, Higgins B, Nicolaides $\mathrm{KH}$. The value of ultrasound in the prediction of successful induction of labor. Ultrasound Obstet Gynecol. 2004;24(5):538-49.[Crossref] [PubMed]
17. Rovas L, Sladkevicius $P$, Strobel E, Valentin L. Three-dimensional power Doppler ultrasound assessment of the cervix for the prediction of successful induction of labor with prostaglandin in prolonged pregnancy. $\mathrm{J} \mathrm{UI-}$ trasound Med. 2005;24(7):933-9.[Crossref] [PubMed]

18. Roman $H$, Verspyck E, Vercoustre L, et al. The role of ultrasound and fetal fibronectin in predicting the length of induced labor when the cervix is unfavourable. Ultrasound Obstet Gynecol. 2004;23(6):567-73.[Crossref] [PubMed]

19. Parkes I, Kabiri D, Hants Y, Ezra Y. The indication for induction of labor impacts the risk of cesarean delivery. J Matern Fetal Neonatal Med. 2016;29(2):224-8.[Crossref] [PubMed]

20. Ashwal E, Hadar E, Chen R, Aviram A, Hiersch L, Gabbay-Benziv R. Effect of fetal gender on the induction of labor failure rates. $J$ Matern Fetal Neonatal Med. 2017;30(24): 3009-13.[Crossref] [PubMed]

21. Tolcher MC, Holbert MR, Weaver AL, et al. Predicting cesarean delivery after induction of labor among nulliparous women at term. Obstet Gynecol. 2015;126(5):1059-68.[Crossref] [PubMed] [PMC]

22. Levine LD, Downes KL, Parry S, Elovitz MA, Sammel MD, Srinivas SK. A validated calculator to estimate the risk of cesarean after induction of labor with an unfavorable cervix [published correction appears in Am J Obstet Gynecol. 2018 May 22:]. Am J Obstet Gynecol. 2018;218(2):254.e1-254.e7.[Crossref] [PubMed] [PMC]

23. Harrell Jr, Frank E. Regression modelling strategies: with applications to linear models, logistic and ordinal regression, and survival analysis. Springer, 2015.[Crossref] 